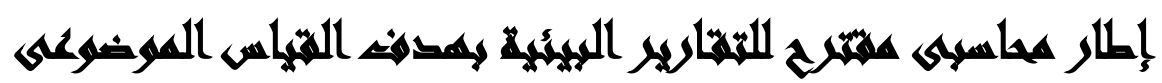 اللأساء المالهي
}

[rv]

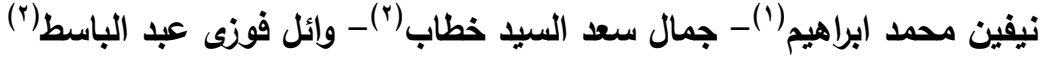

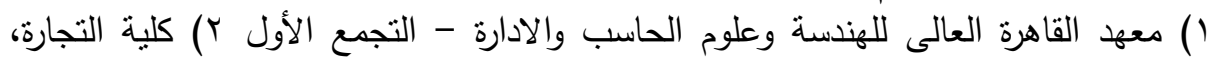
جامعة عين شمس مهن

\section{المستخلي (المه}

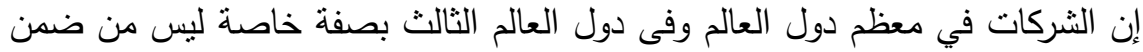

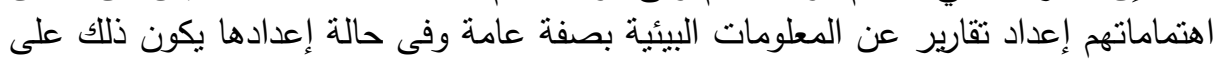

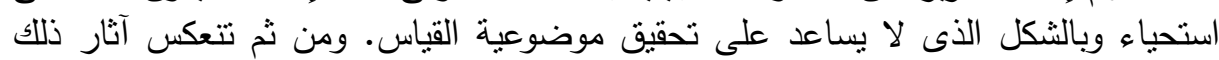

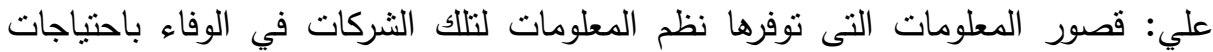
متخذي القرارات وبصفة خاصة فيما يتعلق بالمعلومات البيئية.

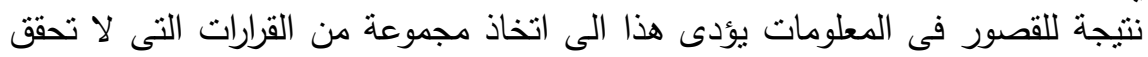

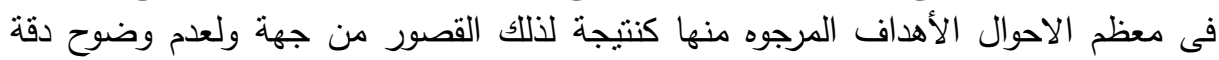

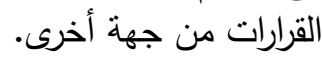
ومن ثم فإن الهدف الرئيسي للاراسةهو "محاولة وضع إطار محاسبي مقترح للتقارير

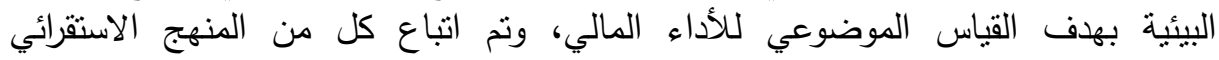

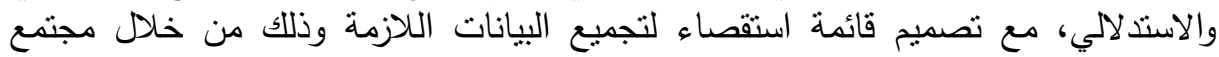

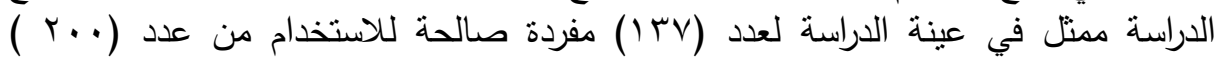

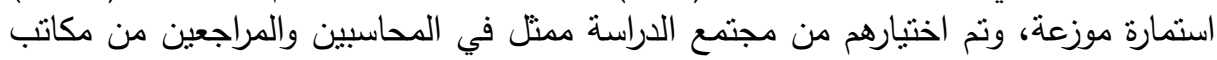

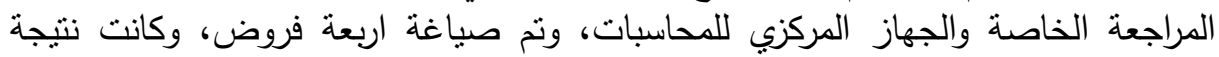

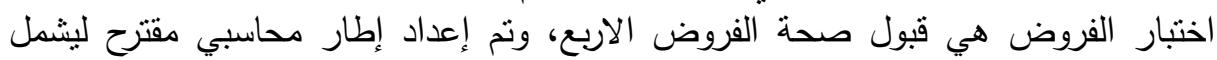

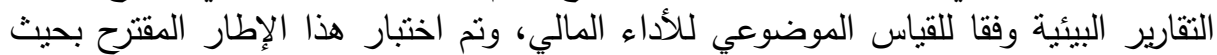

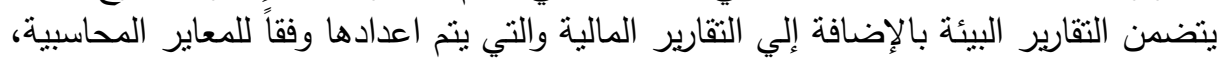

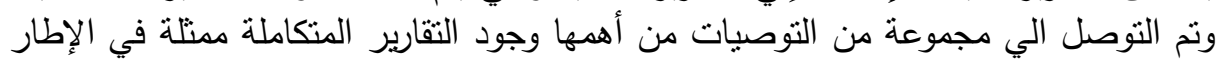

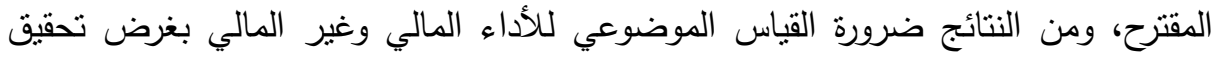

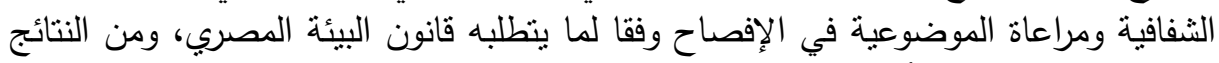
الهامة ضرورة تبويب الأداء البيئي في التقارير المتكاملة. 


\section{xaradl}

أصبحت القضايا المرتبطة بالبيئة ذات صلة أكبر بالمؤسسات، على اختلاف أثنكالها

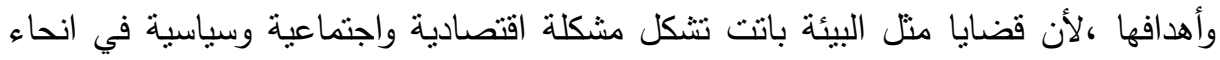

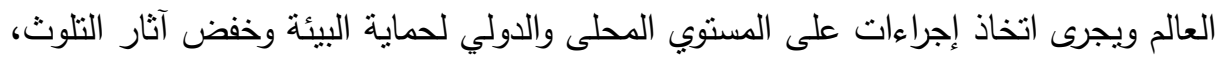
(Elena Dobre 2015) بمعلومات تتعلق بسياسات وأهداف البيئية، والبرامج والتكاليف والمنافع المنصلة بتتفيذ هذه

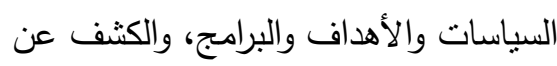
المخاطر البيئية، واتخاذ الترتيبات لتجنبها (Juliana 2012)، ولمعرفة هذه المعلومات

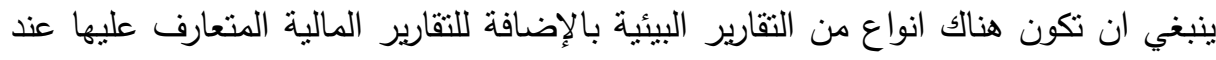
الإفصاح بحيث تشمل هذه الثقارير (Giovanna 2015) المعلومات البيئية مع ربطها بالأداء المالي وبحيث يكون هناك اطار يتم اقتراحه ويهدف هذا الاطار الي القياس الموضوعي للأداء

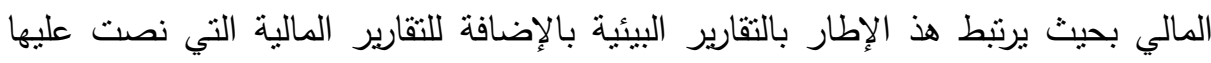
المعايير المحاسبية.

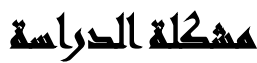

توجد فجوة بحثية نتيجة الاهتمام بإعداد التقارير المالية وفقا للمعايير المحاسبية مع عدم

الاهتمام بالتقارير البيئية خاصة وان العديد من الثركات المؤثرة سلبا في البيئة نتيجة التلوث

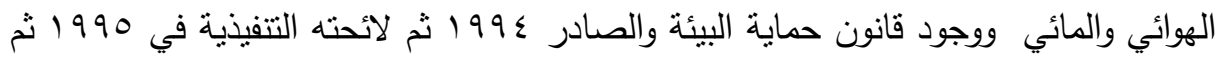

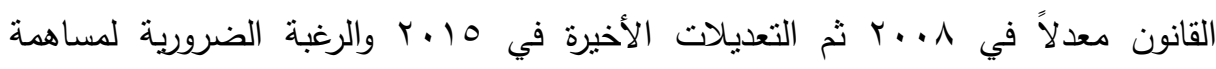
الثركات في المسئولية الاجنماعية وبداية الأنفاق لحماية البيئة الداخلية وكذا البيئة الخارجية

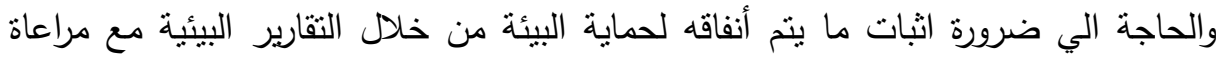

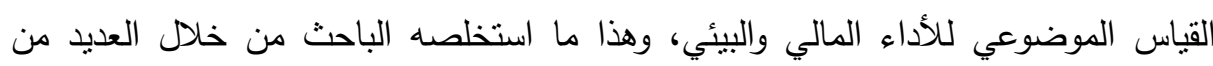

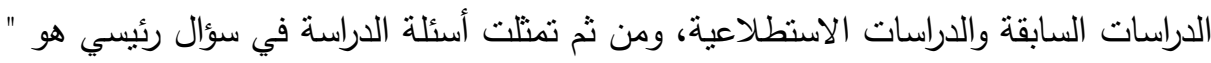


هل يمكن وضع إطار محاسبي مقترح للتقارير البيئية بهدف القياس الموضوعي للأداء المالي؟ ويستمد من هذا السؤال مجموعة من الأسئلة الفرعية النالية:

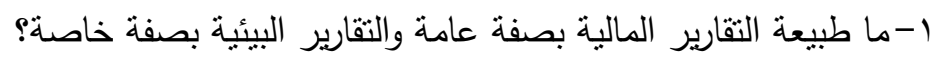

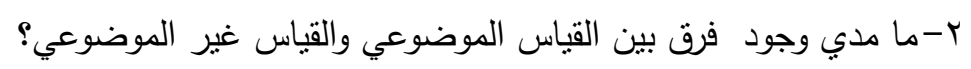

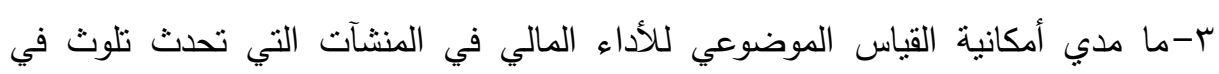
البيئة؛ ع-ما أمكانية وضع تصور لمحتوي إطار محاسبي مقترح للتقارير البيئية بهدف القياس الموضوعي للأداء المالي؟

\section{أهمية التواسمة}

ترجع أهمية الدراسة إلي الأسباب التالية:

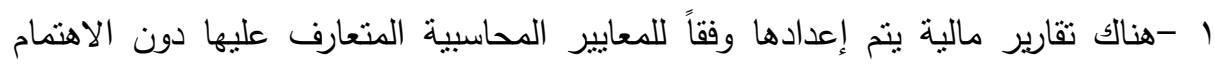

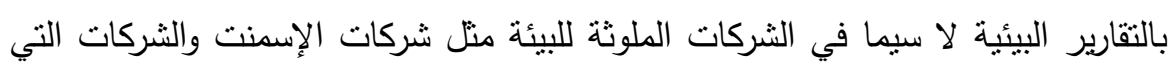

$$
\text { تعمل في البتروكيماويات. }
$$

r - يعتبر الأداء البيئي من منطلبات العصر خاصة وأن هناك العديد من المنشآت الصناعية تحدث تلوث بيئي وهي مطالبة بتتفيذ منطلبات قانون البيئة ومن ثم لابد من أثبات الاداء

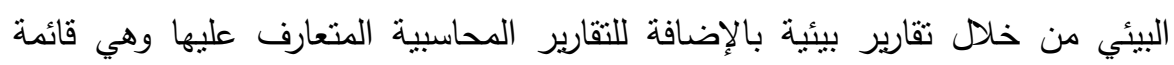
الدخل وقائمة التغيرات في حقوق الملكية وقائمة التدفقات النقدية وقائمة المركز المالي لئي

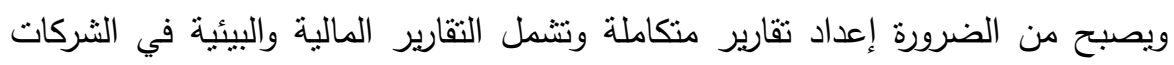
الملوثة للبيئة مثل شركات الإسمنت والثركات التي تعمل في البتروكيماويات.

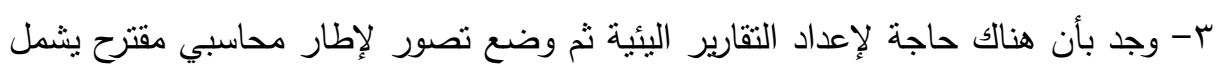
طبيعة التقارير البيئية وفقا للقياس الموضوعي بالإضافة للتقارير المالية التقليدية للشركات

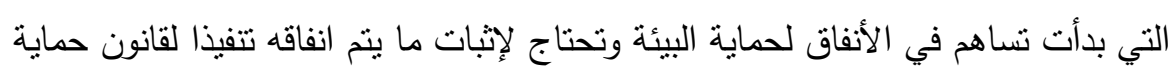




\section{أهساهيت السواسمة}

الههف الرئيسي للاراسة هو "محاولة وضع إطار محاسبي مقترح للتقارير البيئية بهدف القياس الموضوعي للأداء المالي " ويتحقى هذا الهدف من خلال الأهداف الفرعية التالية: ا-بيان طبيعة التقارير المالية بصفة عامة والتقارير البيئية بصفة خاصة. r-بيان وتحديد الفرق بين القياس الموضوعي واقياس غير الموضوعي.

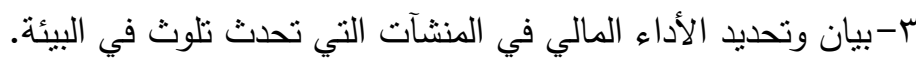
ع-بيان محتوي الإطار المحاسبي المقترح.

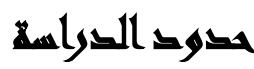

() اقتصرت الدراسة علي اختيار عدد من مكاتب المراجعة الخاصة والمراجعيين في الجهاز

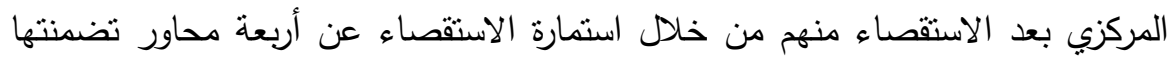
استمارة الاستقصاء ومنها محور تساؤلات عن مضمون الإطار المحاسبي المقترح.

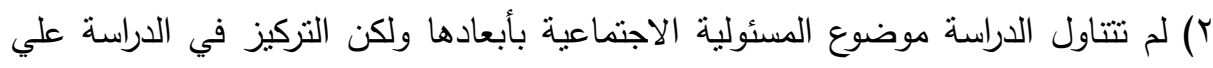
التقارير البيئية ووضعها في إطار متكامل مع التقارير المالية.

\section{ثغوز المهن}

يمكن صياغة فروض الاراسة علي النحو التالي:

الفرض الأول: "يؤدي احتواء الاطار المحاسبي المقترح للتقارير البيئية مع مراعاة القياس

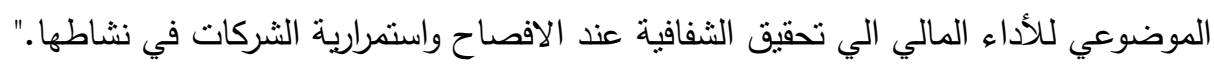
الفرض الثاني:" توجد علاقة ذات دلالة احصائية جوهرية بين ضرورات ومسببات الافصاح

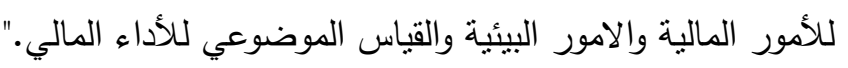

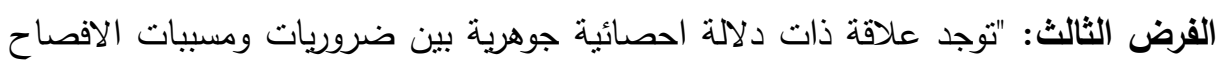

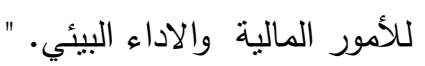


الفرض الرابع: " توجد علاقة ذات دلالة احصائية جوهرية بين الاداء المالي وغير المالي والقياس الموضوعي وفقا للإطار المحاسبي المقترح."

\section{القواساهت المبريعية}

شملت الاراسات المرجعية السابقة العديد منها ومن هذه الدراسات مايلي:

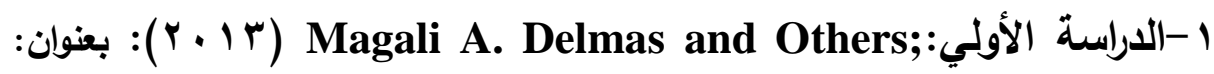

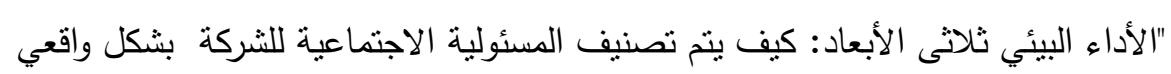

TRIANGULATING ENVIRONMENTAL PERFORMANCE: WHAT DO CORPORATE SOCIAL RESPONSIBILITY RATINGSREALLY CAPTURE?

استهدفت هذه الدراسة محاولة تحديدإذا ما كانت المعلومات عن المسئولية الاجتماعية والتي يتم امدادها عن طريق التظظيمات الرائدة والتي نم تصنيفها الي عدد اقل للأبعاد المتميزة والتني تشمل اعتبارات جوهرية للمسئولية الإجتماعية للشركات وما إذا كانت هذه الأبعاد مرتبطة بالأداء المالي ام غير مرتبطة، وتم تحديد المتغيرات المستخدمة في الدراسة علي اساس مراحل

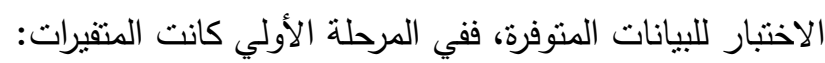
1-الاهتمامات الإجمالية البيئية (الفاقد من المخاطر ، مشاكل الأنبعاثات....) r-القوي الإجمالية (الحملية من النلوث، انظمة الإدارة..) ب- ب-كلفة الدمار الإجمالية ع -الكفاية الاقتصادية

0- إعداد التقارير البيئية،أما متغيرات المرحلة الثنانية هي: الفية

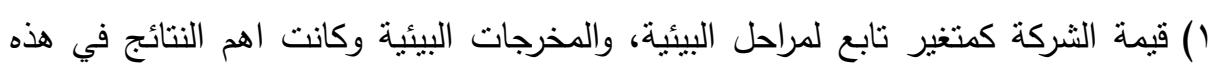
الدراسة هي ان هنا كعاملين متميزين هما: المراحل البيئية وممارسات التتفيذ بمعرفة

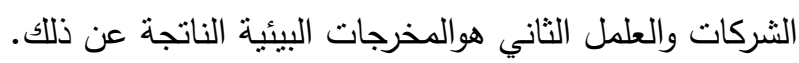
r) وجود ارتباط بين الأداء المالي للشركة والمراحل البيئية ولا توجد هذه العلاقة مع مقاييس لئس المخرجات. 
rena Dobre and Others:الاراسة الثانية بعنوان: "تأثير الأداء البيئي والاجتماعي علي الأداء المالي: الدليل من الوحداتالاقتصادية المدرجة في البورصة الرومانية

The Influence of Environmental and Social Performanceon Financial Performance: Evidence from Romania'sListed Entities

استهدفت الدراسة توفير المعلومات عن كيفية قيام الثركات الرومانية المدرجة بالبورصة بأقرار المؤشرات البيئية والاجتماعية وما إذا كان ذللك يؤثر علي الأداء المالي، وكانت

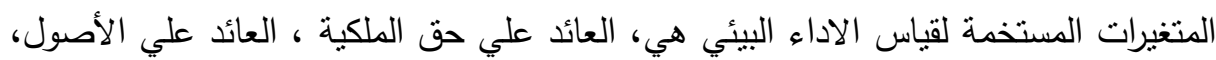

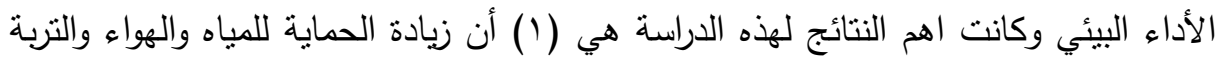

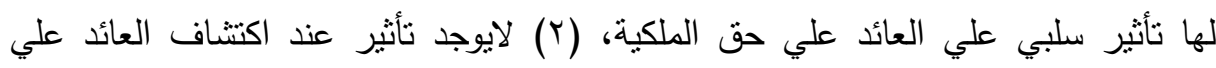

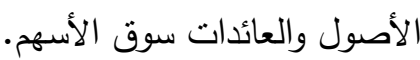

צ - الدراسة الثالثة: Giovanna Campopiano \& Alfredo ( بعنوان: "إعداد تقارير المسئولية الاجتماعية للشركات: تحليل محتوي التقارير للثركات العائلية وتلأك غير العائلية

Corporate Social Responsibility Reporting: A Content Analysisin Family and Non-family Firms"

الهذف: من الدراسة هو اختبار كيفية تأثير الثركات العائلية علي إعداد التقارير الخاصة بالمسئولية الاجتماعية وذللك وفقا للنظرية التنظيمية مقارنا بالثركات غير العائلية، ومن نتائج ( ) تبين ان الثركات العائلية تتشر وتفصح عن تقارير متتوعة بشكل كبير مقارنة بالثركات

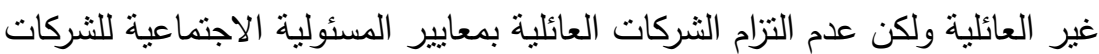
r) تختلف الثركات العائلية عن الثركات غير العائلية في نوع محتوي نقارير المسئولية

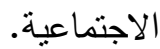
بالإضافة الي العديد من الدراسات السابقة العربية والأجنبية. 


\section{الاجبراءاهي المنهجية}

اشتملت الاراسة اربعة فصول: تضمنت الفصول الثلاثة الإطار العام للاراسة ثم شمل الفصل الثاني والثالث طبيعة التقارير البيئية والفرق بينها وبين التقارير المالية التقليدية ومفهم ومتطلبات القياس الموضوعي للأداء المالي اما الفصل الرابع شمل الدراسة الميدانية وتم تقسيم لتئه

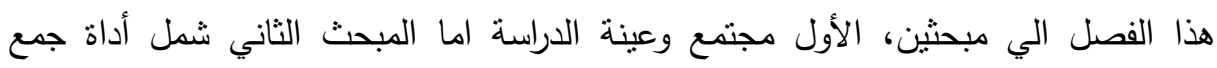
البيانات واختبار فروض الدراسة.

\section{مشور الصواسما}

(1) اقتصر مجتمع وعينة الدراسة علي مراقبي الحسابات في الجهاز المركزي للمحاسبات ومكاتب المراجعة الخاصة بغرض جمع البيانات اللازمة من خلال نوزيع استمارات

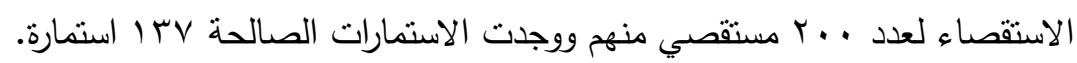

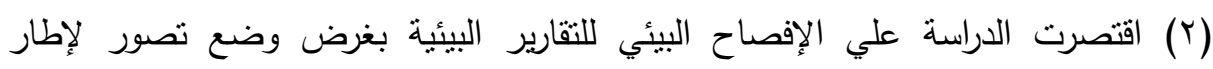
محاسبي مقترح بهذا الثأن (r) لم تتتاول الدراسة المسئولية الاجتماعية للشركات ولا طبيعة المساهمات في التخلص من التلوث البئي.

\section{الأسلوبي المهثيه وهماتر المهيه}

اعتمدت منهجية الدراسة على المنهج الاستقرائي والمنهج الاستتباطي والذى يقوم على الـى

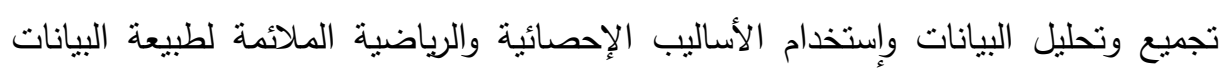

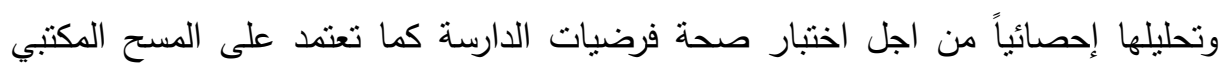
للاستفادة من الكتب والدوريات العلمية فى بناء الإطار النظرى.

\section{هماتر بمع المهيلاهيد}

تم تصميم استمارة استقصاء من أجل تجميع البيانات اللازمة للاراسة، وتم توزيع

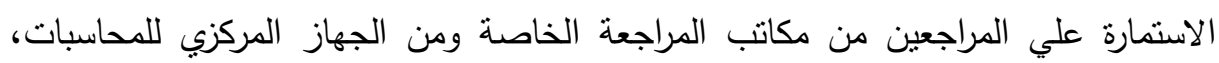

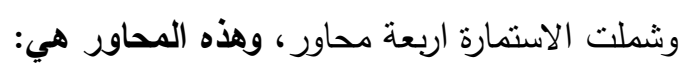

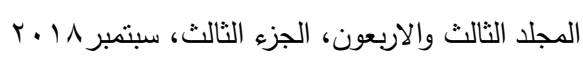


المحور الأول: ضرورة ومسببات الافصاح للأمور المالية وغير المالية والمرنبطة بالبيئية المحور الثاني: ضرورات القياس الموضوعي وعلاقة ذلك بالقياس غير الموضوعي. المحور الثالث: الأداء المالي وغير المالي ونظرة لبطاقة الأداء المنوازن المحور الرابع: محتويات الاطار المحاسبي المقترح للتقارير البيئية وعلاقتها بالأداء المالي. تقييم استمارة الاستقصاء: قام الباحث بتحديد درجة الثبات والمصداقية للدراسة باستخدام معامل الفا كرونباخ، ويتضح من الجدول رقم (1)، أن درجة الثنات 9 (9, · وهي درجة مرتفعة، وانعكس ذلك على درجة المصداقية التي بلغت 901, • وهي درجة ممتازة. مما يدل على أن القائمة تتسم بالصدق والثبات، وهكذا تعثبر القائمة صالحة لجمع البيانات. والجدول رقم (1) قيم معاملات الثبات والصدق. بات والهن

الجدول رقم( ) : يوضح معامل الثبات والمصداقية

\begin{tabular}{|c|c|c|}
\hline 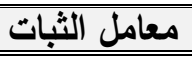 & معامل الصدق & الابعاد \\
\hline$\cdot, \mathrm{r})$. & $\cdot, \wedge \varepsilon \Gamma$ & ضرورة ومسببات الافصاح للمور المالية وغير المالية \\
\hline$\cdot, \Lambda) \leqslant$ & $\cdot, 9 \cdot Y$ & القياس وأهميته الموضوعي وغبر الموضوع \\
\hline$\cdot$, , AOr & $\cdot, 9 r \mu$ & الآداء المالي وغير المالي ونظرة لبطاقة الداء المتوازن \\
\hline$\cdot, \wedge \vee \wedge$ & $\cdot, 9 \mu \mathrm{V}$ & محتويات الاطار المحاسبي المقترح. \\
\hline$\cdot, 919$ &., 901 & الإجمالى \\
\hline
\end{tabular}

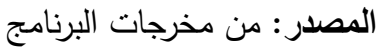

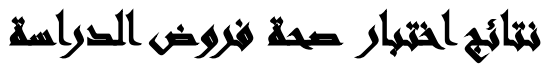

اختبار صحة الفرض الأول: يؤدي احتواء الاطار المحاسبي على الاداء البيئي وفقاً للقياس الموضوعي للأداء المالي وغير المالي الي تحقيق الثفافية عند الافصاح واستمرارية الشركات في نشاطها. يوضح الجدول رقم (Y) مدى الموافقة على أن احتواء الاطار المحاسبي على الاداء البيئي ووفقاً للقياس الموضوعي للأداء المالي يؤدي الي تحقيق الثفافية عند الافصاح واستمرارية الثركات في نشاطها. 
جدول رقم(r): مدى الموافقة على أن احتواء الاطار المحاسبي على الاداء البيئي

\begin{tabular}{|c|c|c|c|c|c|c|c|}
\hline \multicolumn{2}{|c|}{ فتثرة الثقة } & \multirow[b]{2}{*}{ مستولةي } & \multirow[b]{2}{*}{ قيمة ت } & \multirow[b]{2}{*}{ الاتجاه } & \multirow[b]{2}{*}{ النسبي } & \multirow[b]{2}{*}{ المعياري } & \multirow[b]{2}{*}{ المرجح } \\
\hline الحد الأعلى & الألدنى & & & & & & \\
\hline$\varepsilon, r q$ & $\varepsilon, \cdot \varepsilon$ & $\cdot, \cdots$ & $7 \pi, v$. & موافق & $\Delta r, \varepsilon$ & $\cdot, \mathrm{VT}$ & $\varepsilon, 1 V$ \\
\hline
\end{tabular}

يتضح من بيانات الجدول رقم (Y) أن آراء عينة الدراسة ايجابية حيث ان الوزن النسبي

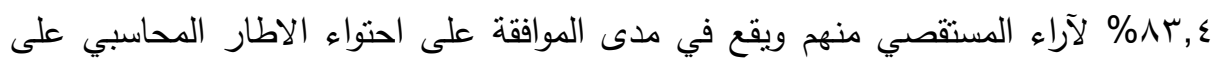

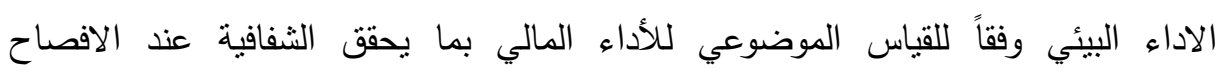

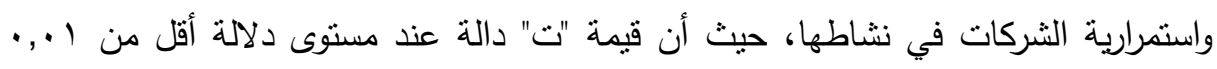

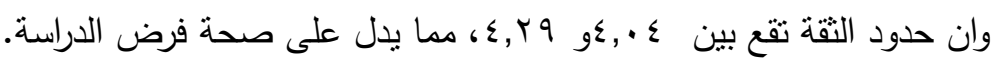
اختبار صحة الفرض الثاني: " توجد علاقة ذات دلالة احصائية جوهرية بين ضرورات ومسبيات الافصاح للأمور المالية والامور البيئية والقياس الموضوعي للأداء المالي."

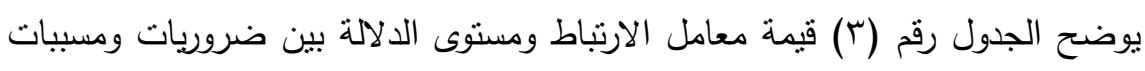

الافصاح للأمور المالية وغير المالية في الامور البيئية والقياس الموضوعي لئية للأداء المالي.

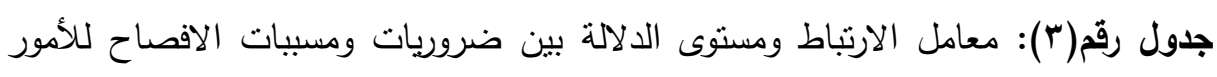

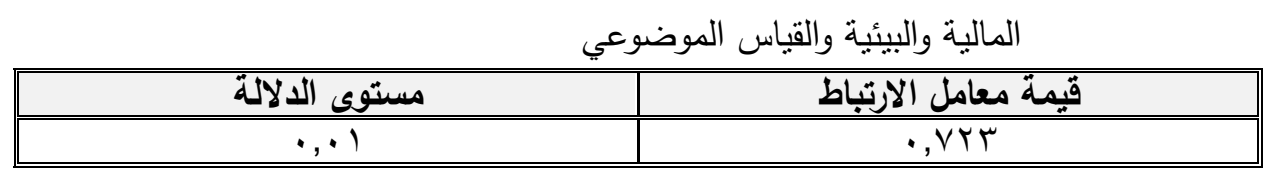

يتضح من بيانات الجدول رقم (r) وجود علاقة دالة احصائية جوهرية بين ضروريات

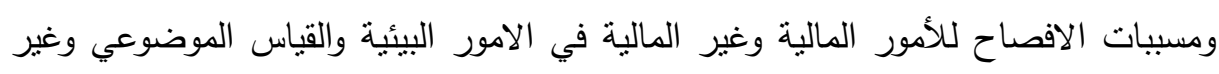

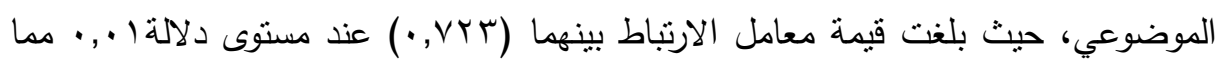
يدل على صحة فرض الدراسة اختبار صحة الفرض الثالث: "توجد علاقة ذات دلالة احصائية جوهرية بين ضروريات ومسبيات الافصاح للأمور المالية والاداء البيئي. "

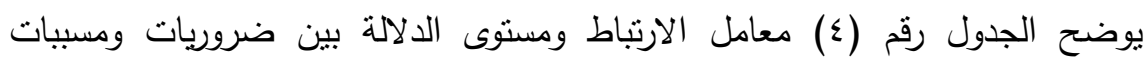
الافصاح للأمور المالية وغير المالية في الامور البيئية والاداء المالي وغير المالي. 
جلول رقم(؛): قيمة معامل الارتباط ومستوى الدلالة بين ضروريات ومسببات الافصاح

\begin{tabular}{|c|c|}
\hline \multicolumn{2}{|c|}{ للأمور البيئية والاداء المالي } \\
\hline مستوى الدلالة & قيمة معامل الارتباط \\
\hline$\cdot, \cdot 1$ & , VOr \\
\hline
\end{tabular}

يتضح من بيانات الجدول رقم (ع )وجود علاقة دالة احصائية جوهرية بين ضروريات

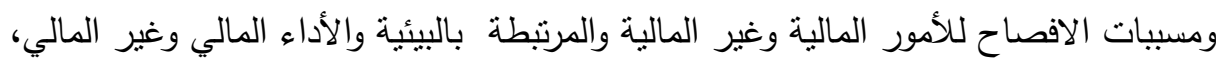

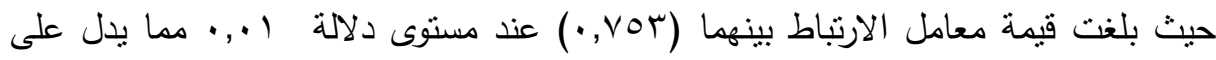

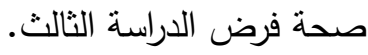
ختبار صحة الفرض الرابع: " توجد علاقة ذات دلالة احصائية جوهرية بين الاداء المالي وغير المالي والقياس الموضوعي وفقا للإطار المحاسبي المقترح."

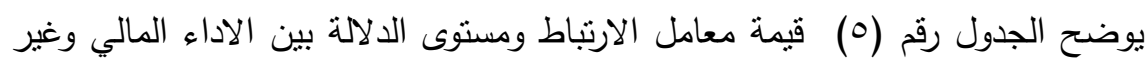
المالي والقياس الموضوعي وغير الموضوعي. جدول رقم(•): قيمة معامل الارتباط ومستوى الدلالة بين الأداء المالي والقياس الموضوعي لموضوعي

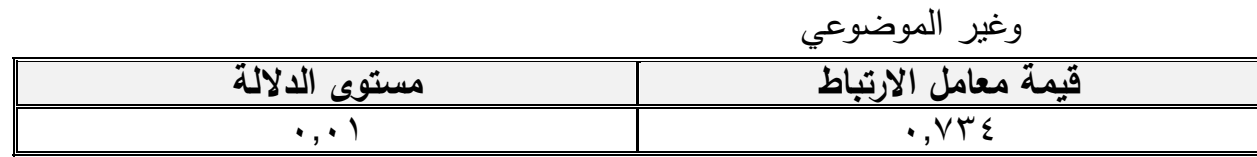

يتضح من بيانات الجدول رقم (0) وجود علاقة دالة احصائياً بين الاداء المالي وغير المالي والقياس الموضوعي وغير الموضوعي، حيث بلغت قيمة معامل الارتباط بينهما

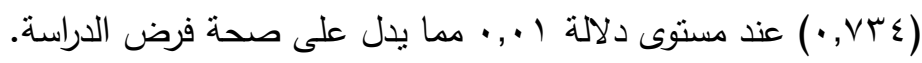

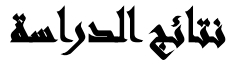

تبين من نتائج اختبار الفروض ما يلي: ا-يؤدي احتواء الاطار المحاسبي على الاداء البيئي وفقاً للقياس الموضوعي للأداء المالي وغير المالي الي تحقيق الثفافية عند الافصاح واستمرارية الثركات في نشاطها وبذلك الكاهي r-توجد علاقة ذات دالة احصائية بين ضروريات الافصاح للأمور المالية وغير المالية في جميع الموضوعات مع مراعاة القياس الموضوعي وغير الموضوعي لما هو منوقع. 674

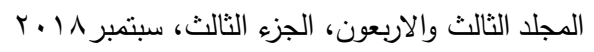


r-توجد علاقة ذات دالة احصائية بين ضروريات الاقصاح للأمور المالية وغير المالية في الموضوعات البيئية والاداء المالي وغير المالي والذي يخص البيئة مثل التوعية.

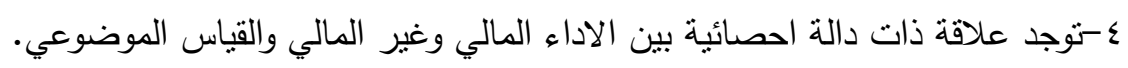
ه-الافصاح يعتبر ضروري وفقا لمعايير المحاسبة المالية بالإضافة الى ما يتطلبه قانون البيئة المصري لاعلام اصحاب المصالح المتعارضه بدور الثركات التي تحدث تلوث في البيئة،

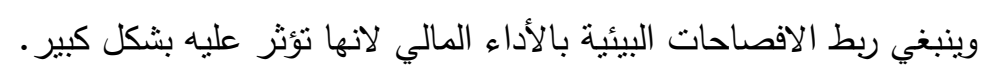

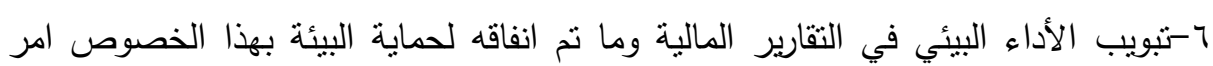

$$
\text { مرغوب فيه للتمشى مع الاتجاه العالمي. }
$$

V-اعداد تقارير للمسئولية الاجتماعية والاداء البيئي منفصله عن التقاهية التهاهير المالية والتي يتم

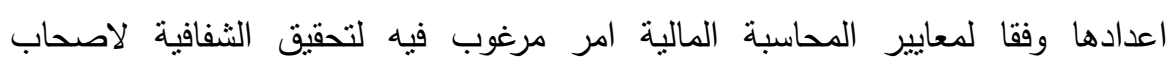

$$
\text { المصالح في المجتمع. }
$$

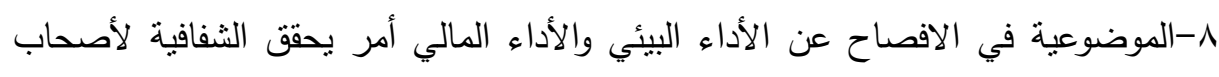

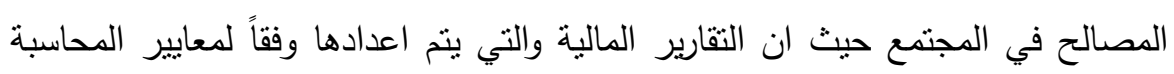

$$
\text { المالية يمكن ان تتضمن الافصاح عن الاداء البيئي بالاضافة للأداء المالي. }
$$

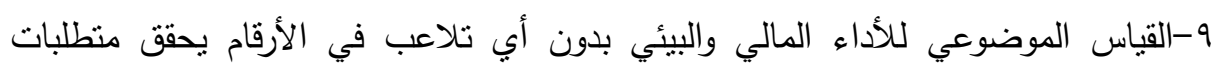

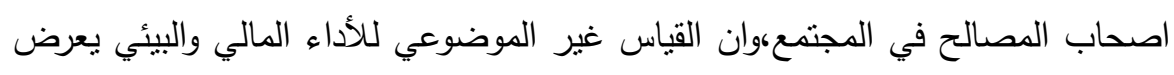

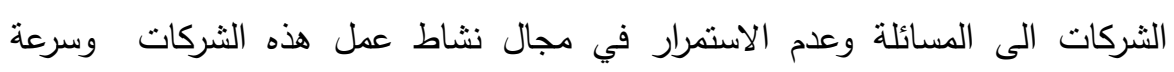

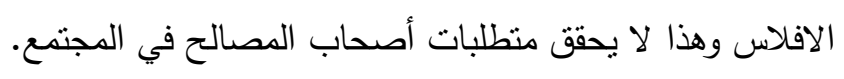

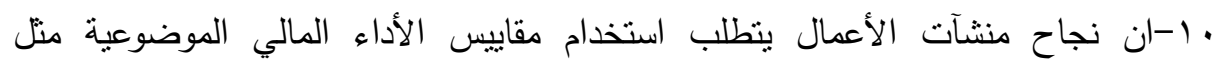

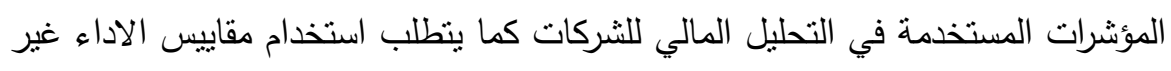
المالي الموضوعية مثل مقاييس متعلقة بالعاملين، والعملاء تطوير وحماية البيئة.وان القياس الموضوعي للأداء المالي والبيئي يحقق الثفافية ومبدأ استمرارية عمل الثركات

$$
\text { المؤثرة في البيئة. }
$$

1) الأداء غير المالي يختص بمدى في قدرة الثركات على حماية العاملين بها والحفاظ على

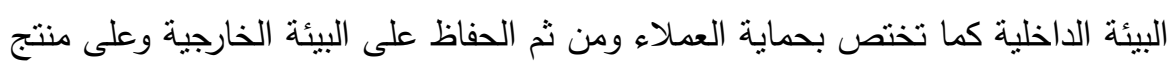

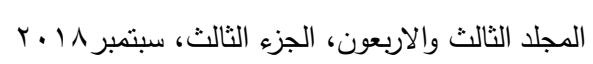


صديق للبيئة والعميل، كما ان الاداء غير المالي يختص بقدرة الثركات على التطوير والنمو ليحافظ على البيئة الداخلية والخارجية. r ا-مقاييس الأداء المالي تختلف عن مقاييس الأداء البيئي وكلاها مطلوب لتحقيق الثفافية عند تحليل مضمون ومحتويات التقارير المالية والبيئية.

\section{اللزوسيايت}

$$
\text { يوصي الباحث بما يلي: }
$$

• أن تتضمن التقارير المالية الأداء البيئي لتحقيق الثفافية عند الافصاح لخدمة أصحاب لواب

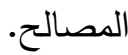
• أن تكون التقارير المالية وتقارير الأداء البيئي منفصلة وحسب رغبة إدارة المنشأة لتحقيق الثفافية عند الافصاح بغرض الاستمرارية للشركات في نشاطتها.

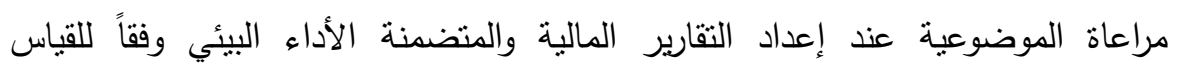
الموضوعي للأداء المالي ولتحقيق الثفافية عند الافصاح المالي والبيئي.

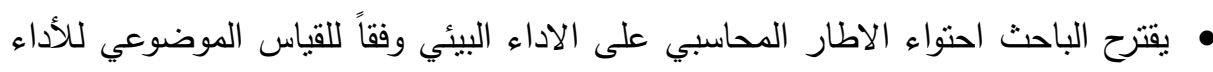

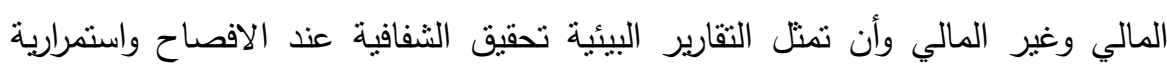

$$
\text { الثركات في نشاطها }
$$

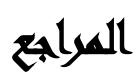

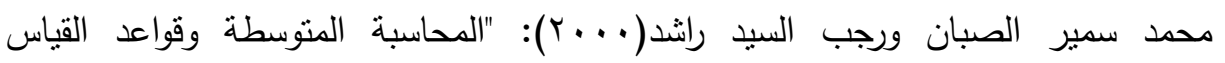

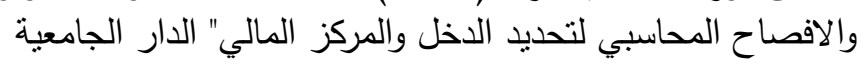

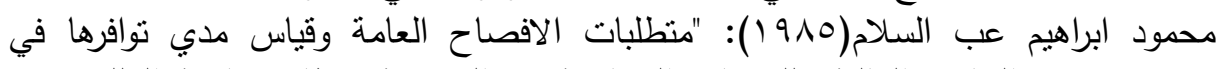

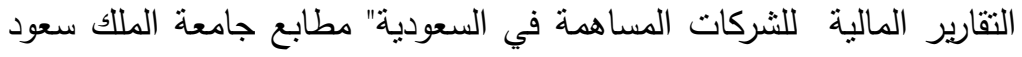

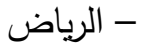

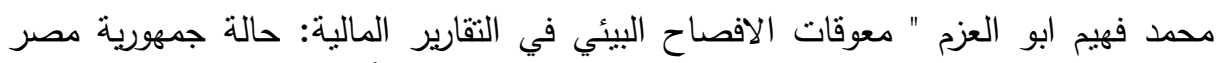

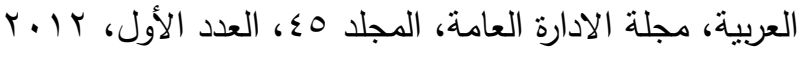




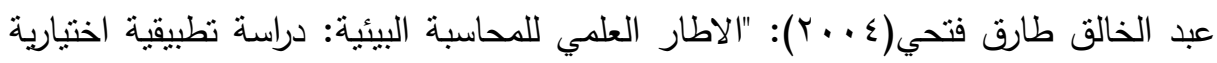
علي القطاع الصناعي ، رسالة دكتوراه - جامعة القاهرة.

CHEEW. CH OW, AND WIMA . VaNDERS T E D E, (2006): "The Use and Usefulness of Nonfinancial Performance Measures, M A N A GEMEN T A C C O U N T IN G Q U A R T E R L Y S P R I N G, V O L. 7, N O . 3

C. Trumpp J. Endrikat C. Zopf E. Guenther , (2015): Definition, Conceptualization, and Measurement of Corporate Environmental Performance: A Critical Examination of A Multidimensional Construct", J Bus Ethics, 126

Elena Dobre $\dagger$, Georgiana Oana Stanila and Laura Brad, (2015): "The Influence of Environmental and Social Performance on Financial Performance: Evidence From Romania's Listed Entities, Sustainability No.7,

Griffin, Jennifer J; Mahon, John F.(1997): “The Corporate Social Performance and Corporate Financial Performance Debate:,Business and Society Mar ; 36, 1

-Giovanna Campopiano Alfredo De Massis, (2015): J Bus Ethics Corporate Social Responsibility Reporting: A Content Analysisin Family and Non-Family Firms"J Bus Ethics 129

Juliana Bonomi Santos And Luizarturledurbrito, (2012): "Toward A Subjective Measurement Model For Firm Performance,,V. 9, Special Issue, Art. 6, , May

Jyrki Niskanen and Terhi Nieminen,(2001): "The Objectivity ofcorporate environmental Reporting:Astudy of Finnish Listedfirms' Environmentaldisclosures", Business Strategy and The Environment 10.

Lonel-Alin Ienciu ,(2012): The Relationship Betweenenvironmental Reporting Andcorporate Governance Characteristicsof Romanian Listed Entities", Accounting And Management Information Systems, Vol. 11, No. 2.

$$
\text { المجلد الثالث والاربعون، الجزء الثالث، سبتمبر 1 ـ r }
$$


Luke Alan Sandham, Felicity Van Der Vyver And Francois Pieter Retief, (2013): September, The Performance of Environmental Impactassessment in The Explosives Manufacturingindustry In South Africa", Journal of Environmental Assessment Policy and Management, Vol. 15, No. 3

Laura Sierra, Anazorio, and María A. García-Benau, (2013): Sustainable Development and Assurance Ofcorporate Social Responsibility Reports Published Byibex-35 Companies", Corporate Social Responsibility And Environmental Management, No.20.

Magali A. Delmas;Droretzion; and Nicholas Nairn-Birch, (2013): Triangulating Environmental Performance: What Do Corporate Socialresponsibility Ratings Really Capture?,Tbe Academy of Management Perspectives, , Vol. 27, No. 3,

Peter A Stanwick; Sarah D Stanwick, (1998): The Relationship Between Corporate Social Performance And Organizational Size,Journal of Business Ethics; Jan 1998; 17, 2

United States Government Accountability Office,(2015): December, "Information Quality Act:Actions Needed To Improve Transparency and Reporting of Correction Requests". 0

1accounting and Management Information Systems, Vol. 11, No. 2. 
نيفين محمد إبراهيم وآخرون

\title{
A SUGGESTED FRAMEWORK FOR ENVIRONMENTAL REPORTS TO REACH THE OBJECTIVITY MEASUREMENTS OF THE FINANCIAL PERFORMANCE
}

Ibrahim, Neveen, M. ${ }^{(1)}$; Khatab, G. S. ${ }^{(2)}$ and Abdel Basit,.W. F. ${ }^{(2)}$ 1) Cairo Higher Institute For Engineering, Computer, and Management Science, The First Assembly 2) Faculty of Commerce, Ain Shams University

\begin{abstract}
Environmental issues became a critical matter in all over the world specially in the less development countries. While applying the Environmental Law in Egypt and corporations starts to implement the Law, it is important for corporations to report all items related to cost and expenditure for protecting the environment, whether internal or external environments. The main objective of this paper is to provide a suggested Accounting Framework for environmental reports to reach the objectivityof measurements of the financial performance. A questionnaire had been developed and distributed to Accountants in the private accounting firms and in the governmental accounting offices and the valid number of questionnaires was 137 out of 200. The most important results indicate that the suggested Accounting Framework must include the environmental reports according to the objectivity measurements for the financial performance. Recommendation has been presented.
\end{abstract}

Key Words: Financial performance, Environmental performance, objectivity, Financial reports, Environmental reports. 